Wilfrid Laurier University

Scholars Commons @ Laurier

2006

\title{
Dynamic Coupling of Piezoelectric Effects, Spontaneous Polarization, and Strain in Lattice-Mismatched Semiconductor Quantum-Well Heterostructures
}

\author{
Morten Willatzen \\ University of Southern Denmark \\ Benny Lassen \\ University of Southern Denmark \\ L.C. Lew Yan Voon \\ Wright State University \\ Roderick V.N. Melnik \\ Wilfrid Laurier University, rmelnik@wlu.ca
}

Follow this and additional works at: https://scholars.wlu.ca/math_faculty

\section{Recommended Citation}

Willatzen, Morten; Lassen, Benny; Yan Voon, L.C. Lew; and Melnik, Roderick V.N., "Dynamic Coupling of Piezoelectric Effects, Spontaneous Polarization, and Strain in Lattice-Mismatched Semiconductor Quantum-Well Heterostructures" (2006). Mathematics Faculty Publications. 40.

https://scholars.wlu.ca/math_faculty/40

This Article is brought to you for free and open access by the Mathematics at Scholars Commons @ Laurier. It has been accepted for inclusion in Mathematics Faculty Publications by an authorized administrator of Scholars Commons@ Laurier. For more information, please contact scholarscommons@wlu.ca. 


\title{
Dynamic coupling of piezoelectric effects, spontaneous polarization, and strain in lattice-mismatched semiconductor quantum-well heterostructures
}

\author{
M. Willatzen ${ }^{\mathrm{a})}$ and B. Lassen \\ Mads Clausen Institute, University of Southern Denmark, Grundtvigs Allé 150, \\ DK-6400 Sønderborg, Denmark \\ L. C. Lew Yan Voon \\ Department of Physics, Wright State University, 3640 Colonel Glenn Highway, Dayton, Ohio 45435 \\ R. V. N. Melnik \\ Wilfrid Laurier University, 75 University Avenue West, Waterloo, Ontario N2L 3C5, Canada
}

(Received 1 February 2006; accepted 11 May 2006; published online 17 July 2006)

A static and dynamic analysis of the combined and self-consistent influence of spontaneous polarization, piezoelectric effects, lattice mismatch, and strain effects is presented for a three-layer one-dimensional AlN/GaN wurtzite quantum-well structure (with $\mathrm{GaN}$ as the central quantum-well layer). It is shown that, contrary to the assumption of Fonoberov and Balandin [J. Appl. Phys. 94, 7178 (2003); J. Vac. Sci. Technol. B 22, 2190 (2004)], even in cases with no current transport through the structure, the strain distributions are not well captured by minimization of the strain energy only and not, as is in principle required, the total free energy including electric and piezoelectric coupling and spontaneous polarization contributions. Furthermore, we have found that, when an ac signal is imposed through the structure, resonance frequencies exist where strain distributions are even more strongly affected by piezoelectric-coupling contributions depending on the amount of mechanical and electrical losses in the full material system. (C) 2006 American Institute of Physics. [DOI: 10.1063/1.2214361]

\section{INTRODUCTION}

GaN/AlN quantum-confined wurtzite heterostructure material combinations are promising candidates for optical and electronic-device applications. Hence, it is necessary to understand the complicated coupling phenomena involving mechanical strain, electrical effects including spontaneous polarization, piezoelectric effects, and lattice mismatch present in GaN/AlN wurtzite heterostructures. These coupling phenomena are expected to have important implications for conduction- and valence-band dispersion relations and associated eigenstates as well as optoelectronic properties. ${ }^{1-3}$ It must be emphasized that the above fields and eigenstates are found, in principle, by self-consistently solving the Schrödinger equation, Maxwell-Poisson equation, and Navier's equations along with constitutive relations on stress and electric displacement relevant to the wurtzite crystal structure in consideration.

In this work, we take a first step along these lines by solving analytically and self-consistently (under static and dynamic conditions) the Maxwell-Poisson equation and Navier's equations accounting for lattice mismatch effects in the effective strain tensor ${ }^{4}$ in the absence of free-carrier charge distributions. The latter condition allows the electromechanical fields to be determined without solving simultaneously the Schrödinger equation with others since electronic eigenstates, in this case, do not appear in terms in the Maxwell-Poisson equation. The reasons for the above-

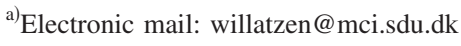

described calculations are threefold. First, some of the most recent and sophisticated calculations of the electronic states in wurtzite AlN/GaN quantum dots ${ }^{1,2}$ have completely ignored the fully coupled model of the piezoelectric effect. Nevertheless, there are apparently conflicting results regarding the need for a fully coupled model even for quantumwell structures. ${ }^{3,5}$ Thus, Jogai et al. ${ }^{5}$ argued that the internal electric field can be as high as $5 \mathrm{MV} / \mathrm{cm}$ for $\mathrm{AlGaN} / \mathrm{GaN}$ and, consequently, the difference between a full coupled model and a semicoupled model can be as high as 60\%. On the other hand, calculations just published by Christmas et $a .^{3}{ }^{3}$ found only a $2 \%$ error in the internal fields for $\mathrm{InGaN} / \mathrm{GaN}$. We, therefore, reanalyze the quantum-well problem using an analytical model where we generalize the above work to include internal fields in both layers. Finally, we point out that all of the above-referenced works were performed under static conditions while many applications (e.g., laser diodes) would require ac response; hence, we consider the latter case here. For simplicity, we will refer to the AlN/GaN material system though we have carried out equivalent calculations for the $\mathrm{AlGaN} / \mathrm{GaN}$ and $\mathrm{InGaN} / \mathrm{GaN}$ systems and relevant results will be mentioned below.

\section{THEORY}

The governing equations for a lattice-mismatched heterostructure consisting of two wurtzite materials $(\mathrm{GaN}$ and AlN) accounting for piezoelectric effects and spontaneous polarization read 


$$
\begin{aligned}
& \mathbf{T}=\mathbf{c S}-\mathbf{e E}, \\
& \mathbf{D}=\epsilon \mathbf{E}+\mathbf{P}^{\mathrm{sp}}+\mathbf{e S}, \\
& \nabla \cdot \mathbf{T}=\rho_{d} \frac{\partial^{2} \mathbf{u}}{\partial t^{2}}, \\
& \nabla \cdot \mathbf{D}=\rho_{f},
\end{aligned}
$$

where $\mathbf{T}, \mathbf{S}, \mathbf{D}, \mathbf{E}, \mathbf{P}^{\mathrm{sp}}$, and $\mathbf{u}=\left(u_{x}, u_{y}, u_{z}\right)$ are the stress tensor, strain tensor, electric displacement, electric field, spontaneous polarization, and the particle displacement, respectively. The coefficients $\mathbf{c}, \epsilon, \mathbf{e}, \rho_{d}$, and $\rho_{f}$ denote the positiondependent elastic tensor, permittivity tensor, piezoelectric $\mathbf{e}$ tensor, the mass density, and the free-charge carrier density, respectively. The strain $\mathbf{S}$ accounts for lattice mismatch and is defined by ${ }^{4}$

$$
\begin{aligned}
& S_{1}=\frac{\partial u_{x}}{\partial x}-\frac{a(\overrightarrow{\mathbf{r}})-a^{(1)}}{a^{(1)}}, \quad S_{4}=\frac{1}{2}\left(\frac{\partial u_{y}}{\partial z}+\frac{\partial u_{z}}{\partial y}\right), \\
& S_{2}=\frac{\partial u_{y}}{\partial y}-\frac{a(\overrightarrow{\mathbf{r}})-a^{(1)}}{a^{(1)}}, \quad S_{5}=\frac{1}{2}\left(\frac{\partial u_{x}}{\partial z}+\frac{\partial u_{z}}{\partial x}\right), \\
& S_{3}=\frac{\partial u_{z}}{\partial z}-\frac{c(\overrightarrow{\mathbf{r}})-c^{(1)}}{c^{(1)}}, \quad S_{6}=\frac{1}{2}\left(\frac{\partial u_{x}}{\partial y}+\frac{\partial u_{y}}{\partial x}\right),
\end{aligned}
$$

where $a(\overrightarrow{\mathbf{r}})$ and $c(\overrightarrow{\mathbf{r}})$ are the lattice constants at position $\overrightarrow{\mathbf{r}}$, while $a^{(1)}$ and $c^{(1)}$ denote the lattice constants of AIN (a convenient choice for a GaN quantum-well layer grown along the [001] direction and sandwiched between two layers of AlN).

In the following, we examine whether piezoelectric effects and spontaneous-polarization effects alter the strain distribution. It was assumed in previous papers by Fonoberov and Balandin ${ }^{1,2}$ that the strain distribution is determined by minimizing the elastic energy only, i.e., not accounting for feedback coupling from piezoelectric effects and spontaneous polarization on strain. In order to simplify the description, we assume that all fields are functions of the growth direction $(z)$ only, i.e., we disregard dependencies on the $x$ and $y$ coordinates. In this way, analytical results can be found for the cases with (a) electric displacement constant through the heterostructure and constant in time, and (b) electric displacement constant through the structure but harmonic in time. It should be pointed out that although only $z$ dependencies are included, the important contribution from the static mechanical Poisson coupling in the lateral directions is accounted for. In the present analysis, surface stresses are neglected. This means that the model is expected to work well particularly in cases where the AlN layer thicknesses are considerably larger than the GaN layer such that surfacestress contributions ${ }^{6}$ at the AIN interfaces with the embedding material (often vacuum) are negligible.

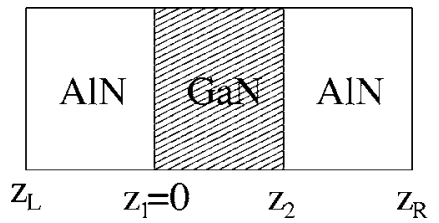

FIG. 1. Schematics of the three-layer quantum-well structure considered in the present work.

\section{A. Constant electric displacement in space and time}

In the static case without free-charge carriers in the heterostructure, Maxwell's equation [Eq. (4)] for the onedimensional case leads to a constant electric displacement in space. Navier's equations is given by

$$
\frac{\partial T_{3}}{\partial z}=\frac{\partial T_{4}}{\partial z}=\frac{\partial T_{5}}{\partial z}=0,
$$

applying in each homogeneous material layer we have

$$
\frac{\partial^{2} u_{x}}{\partial z^{2}}=\frac{\partial^{2} u_{y}}{\partial z^{2}}=\frac{\partial^{2} u_{z}}{\partial z^{2}}=0,
$$

in each layer, respectively. Maxwell's equation requires

$$
\frac{\partial^{2} \phi}{\partial z^{2}}=0,
$$

in each layer, where $\phi$ is the electric potential $(\mathbf{E}=-\nabla \phi)$.

For a three-layer structure, as shown in Fig. 1, boundary conditions at the interface between AlN and the material embedding the three-layer structure are

$$
\begin{aligned}
& T_{4}=T_{5}=0, \\
& D_{z}=D, \\
& T_{3}=\mathcal{Z} S_{3},
\end{aligned}
$$

corresponding to positions $z=z_{L}$ and $z=z_{R}$. In Eq. (11), $\mathcal{Z}$ denotes the effective stiffness of the embedding material and it has been assumed that shear stresses in the embedding material are negligible. The boundary conditions at the AlN/GaN interfaces $\left(z=z_{1}\right.$ and $\left.z=z_{2}\right)$ are

$$
T_{3}, T_{4}, T_{5}, \phi, D_{z}, u_{x}, u_{y}, u_{z} \text { continuous. }
$$

In addition, the potential and particle displacements are known to within an arbitrary constant. Hence, we may fix their values such that

$$
u_{x}\left(z=z_{1}\right)=u_{y}\left(z=z_{1}\right)=u_{z}\left(z=z_{1}\right)=\phi\left(z=z_{L}\right)=0,
$$

and choose the coordinate system corresponding to $z_{1}=0$. It is an easy task to solve for the variables involved analytically. The results are

$$
u_{x}=u_{y}=0, \quad \text { everywhere, }
$$

with 


$$
\begin{array}{ll}
u_{z}=\mathcal{A}_{3}+\mathcal{B}_{3} z, & z_{L} \leqslant z \leqslant 0, \\
u_{z}=\mathcal{C}_{3}+\mathcal{D}_{3} z, & 0 \leqslant z \leqslant z_{2}, \\
u_{z}=\mathcal{E}_{3}+\mathcal{F}_{3} z, & z_{2} \leqslant z \leqslant z_{R},
\end{array}
$$

for the z-particle displacement, and

$$
\begin{aligned}
& \phi=\mathcal{A}_{4}+\mathcal{B}_{4} z, \quad z_{L} \leqslant z \leqslant 0, \\
& \phi=\mathcal{C}_{4}+\mathcal{D}_{4} z, \quad 0 \leqslant z \leqslant z_{2}, \\
& \phi=\mathcal{E}_{4}+\mathcal{F}_{4} z, \quad z_{2} \leqslant z \leqslant z_{R},
\end{aligned}
$$

for the electric potential. The coefficients appearing in the above expressions read

$$
\begin{aligned}
& \mathcal{A}_{3}=0, \quad \mathcal{B}_{3}=\frac{e_{33}^{(1)}\left(D-P^{\mathrm{sp}, 1}\right)}{e_{33}^{(1) 2}+\left(c_{33}^{(1)}-\mathcal{Z}\right) \epsilon^{\|, 1}}, \quad \mathcal{C}_{3}=0 \\
& \mathcal{D}_{4}=\frac{c_{33}^{(2)}\left(P^{\mathrm{sp}, 2}-D\right)+2\left[c_{13}^{(2)} e_{33}^{(2)}-c_{33}^{(2)} e_{31}^{(2)}\right] a_{\mathrm{mis}}+e_{33}^{(2)} \mathcal{Z} \mathcal{B}_{3}}{c_{33}^{(2)} \epsilon^{\|, 2}+e_{33}^{(2) 2}}, \\
& \mathcal{F}_{3}=\mathcal{B}_{3}, \\
& \mathcal{D}_{3}=\frac{2 c_{13}^{(2)} a_{\mathrm{mis}}+c_{33}^{(2)} c_{\mathrm{mis}}-e_{33}^{(2)} \mathcal{D}_{4}+\mathcal{Z} \mathcal{B}_{3}}{c_{33}^{(2)}}, \\
& \mathcal{E}_{3}=\left(\mathcal{D}_{3}-\mathcal{F}_{3}\right) z_{2}, \\
& \mathcal{B}_{4}=\frac{\mathcal{Z}-c_{33}^{(1)}}{e_{33}^{(1)} \mathcal{B}_{3}, \quad \mathcal{A}_{4}=-\mathcal{B}_{4} z_{L}, \quad \mathcal{C}_{4}=\mathcal{A}_{4}, \quad \mathcal{F}_{4}=\mathcal{B}_{4},} \\
& \mathcal{E}_{4}=\mathcal{C}_{4}+\left(\mathcal{D}_{4}-\mathcal{F}_{4}\right) z_{2},
\end{aligned}
$$

where superscripts 1 and 2 on parameters refer to values in AlN and GaN, respectively. Similarly, $e_{33}$ and $\epsilon^{\|}$are the "33" component, the parallel-permittivity component, and likewise for other tensor quantities, while $a_{\mathrm{mis}}=\left[a^{(2)}-a^{(1)}\right] / a^{(1)}$,
TABLE I. Contributions to the vertical strain in a given layer with $\mathrm{GaN}$ as buffer or substrate for the first three cases, and with AlN as the substrate for the last one. Parameters are taken from Jogai et al. (Ref. 5) and Christmas et al. (Ref. 3).

\begin{tabular}{lrrr}
\hline \hline & $S_{\text {uncoupled }}$ & \multicolumn{1}{c}{$S_{\text {coupled }}$} & \multicolumn{1}{c}{$S_{\text {spon }}$} \\
\hline $\mathrm{AlN} / \mathrm{GaN}$ & -0.0143 & 0.0026 & 0.0026 \\
$\mathrm{Al}_{0.3} \mathrm{Ga}_{0.7} \mathrm{~N} / \mathrm{GaN}$ & -0.0039 & 0.0004 & 0.0005 \\
$\mathrm{In}_{0.2} \mathrm{Ga}_{0.8} \mathrm{~N} / \mathrm{GaN}$ & 0.0119 & -0.0008 & 0.0001 \\
$\mathrm{GaN} / \mathrm{AlN}$ & 0.0123 & 0.0008 & -0.0014 \\
\hline \hline
\end{tabular}

$c_{\text {mis }}=\left[c^{(2)}-c^{(1)}\right] / c^{(1)}$ are the relative lattice-mismatch values. Observe that when piezoelectric effects are absent, the strain constant $\mathcal{D}_{3}=\partial u_{z} / \partial z=\left[2 c_{13}^{(2)} a_{\text {mis }}+c_{33}^{(2)} c_{\text {mis }}\right] / c_{33}^{(2)}$, i.e., the strain coefficient $S_{3}$ becomes $\left(\partial u_{z} / \partial z\right)-c_{\text {mis }}=2\left(c_{13}^{(2)} / c_{33}^{(2)}\right) a_{\text {mis. }}$. In other words, we obtain the well-known Poisson-ratio effect for a quantum-well layer (of $\mathrm{GaN}$ ) forced to shrink its inplane lattice constant to that of the material layer (AlN) surrounding the quantum well.

We use the same parameters for AlN and GaN as in Refs. 1 and 2. Since $O\left(e_{33}\right)=1$ and $O\left(c_{33}\right)=5 \times 10^{11}$ [here, and in the following, data values are given in Systeme International (SI) units], the influence of piezoelectric effects on strain distributions is very small for small external fields but it increases linearly with the electric field. For example, imposing a large electric field $\Delta E$ of approximately $5 \times 10^{8}$ (depends slightly on material as $D_{4} \neq B_{4}$ ) results in a piezoelectrically induced strain contribution equal to

$$
\Delta S_{3}=\frac{e_{33}^{(2)}}{c_{33}^{(2)}} \Delta E \approx 1 \times 10^{-3},
$$

in $\mathrm{GaN}$ with stress-free conditions $(\mathcal{Z}=0)$ at the $\mathrm{AlN}$ ambedding layer interfaces corresponding to the case where the embedding layer is vacuum. We will also see below, in Table I, that this is the same order of magnitude as the strain due to the internal fields. Compared to the mismatch strain of $2.4 \%$, this is not negligible but certainly not big either. In Fig. 2 (upper plot), we show the induced strain $S_{3}=\mathcal{D}_{3}$
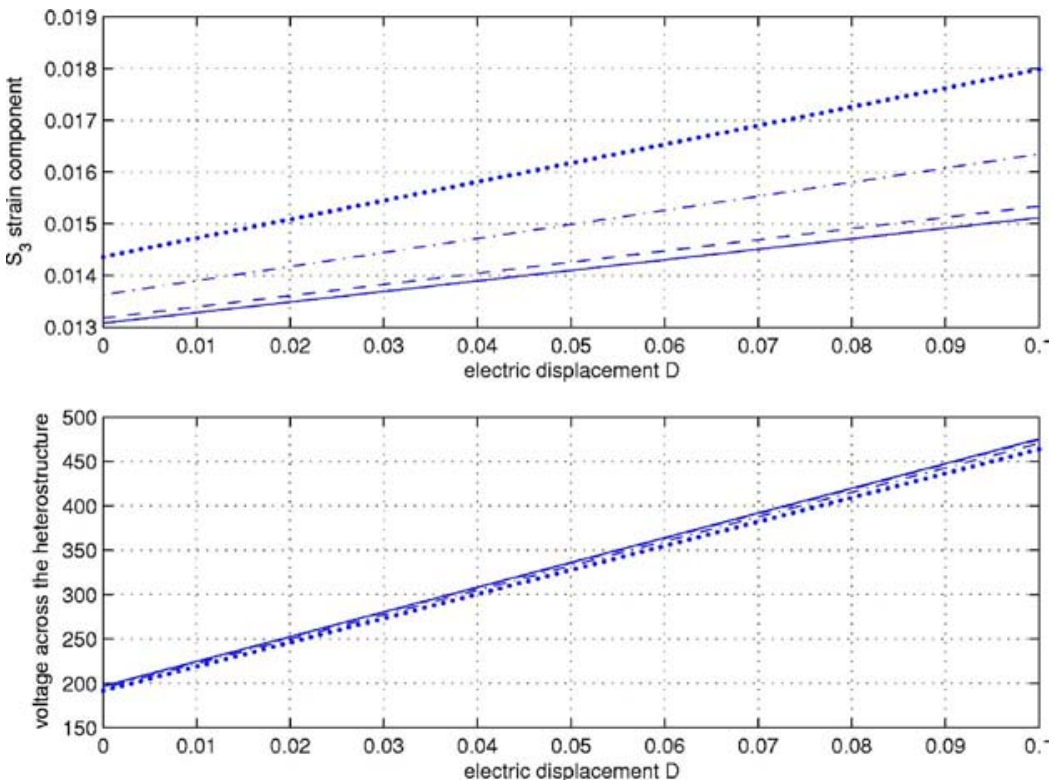

FIG. 2. (Color online) The upper plot shows the strain component $S_{3}$ in the static case as a function of electric displacement $D$ for four different cases of material layers embedding AlN. The lower plot depicts the corresponding voltage across the heterostructure as a function of electric displacement $D$. Line codings are as follows: $\mathcal{Z}=0$ (solid-equivalent to vacuum), $\mathcal{Z}=1$ $\times 10^{10}$ (dashed), $\mathcal{Z}=5 \times 10^{10}$ (dash-dotted), and $\mathcal{Z}=1$ $\times 10^{11}$ (dotted). Figure values are in SI units. 
$-c_{\text {mis }}$ in the GaN layer due to the imposed electric displacement for $D$ values in the range of $0-0.1$ corresponding to various embedding materials $[\mathcal{Z}=0$ (solid-equivalent to vacuum), $\mathcal{Z}=1 \times 10^{10}$ (dashed), $\mathcal{Z}=5 \times 10^{10}$ (dash-dotted), and $\mathcal{Z}=1 \times 10^{11}$ (dotted)]. In Fig. 2 (lower plot), the voltage across the full heterostructure is plotted versus the electric displacement for the same four cases of embedding materials.

However, the previous calculation [Eq. (18)] was for a semicoupled calculation. In a fully coupled model [expressions derived in Eq. (17)], the internal fields can also influence the strain distribution via the indirect effect, one not taken into consideration by Fonoberov et al. ${ }^{1,2}$ In this case, our equations reduce to Eq. (15) of Jogai et al. ${ }^{5}$ and to Eqs. (12) and (13) of Christmas et al. ${ }^{3}$ in the appropriate limits. The most transparent form is that of Jogai et al. ${ }^{5}$ which we rewrite below as [without the two-dimensional (2D) electron gas]

$$
\begin{aligned}
S_{3}= & -\frac{2 c_{13}^{(1)}}{c_{33}^{(1)}} S_{1}+\left\{\frac{2 e_{33}^{(1)}\left[e_{33}^{(1)} c_{13}^{(1)}-e_{13}^{(1)} c_{33}^{(1)}\right]}{c_{33}^{(1)}\left[\epsilon_{\|, 1} c_{33}^{(1)}+e_{33}^{(1)}\right]}\right\} S_{1} \\
& +\frac{e_{33}^{(1)}\left(P^{\mathrm{sp}, \mathrm{GaN}}-P^{\mathrm{sp}, 1}\right)}{\epsilon_{\|, 1} c_{33}^{(1)}+e_{33}^{(1) 2}} \\
\equiv & S_{\text {uncoupled }}+S_{\text {coupled }}+S_{\text {spon }},
\end{aligned}
$$

where we have labeled the three strain terms according to their origin and we choose the $\mathrm{GaN}$ to be a substrate layer and layer " 1 " will be AlN, AlGaN, and InGaN. The relative magnitude of the three strain contributions are given in Table I for the three material compositions: AlN/GaN, $\mathrm{Al}_{0.3} \mathrm{Ga}_{0.7} \mathrm{~N} / \mathrm{GaN}$, and $\mathrm{In}_{0.2} \mathrm{Ga}_{0.8} \mathrm{~N} / \mathrm{GaN}$. To compare to the structure we have adopted in this paper, where the $\mathrm{GaN}$ is the active well region, we have also included (as the last row in Table I) the situation where AlN is the substrate (labeled GaN/AlN). Table I shows that the relative difference between an uncoupled and a coupled calculation is of order $18 \%, 10 \%$, and $7 \%$ for $\mathrm{AlN}, \mathrm{AlGaN}$, and $\mathrm{InGaN}$, respectively. But we note that the spontaneous polarization contribution adds another $18 \%, 13 \%$, and $0.8 \%$. This simple analysis, not previously discussed in the literature, explains the apparent contradiction between the results of Jogai et al. ${ }^{5}$ and Christmas et al. ${ }^{3}$ Thus, the extremely large coupling reported by Jogai et al. ${ }^{5}$ for $\mathrm{AlGaN}$ is here seen to be, in fact, due to combined influence of piezoelectric coupling and spontaneous polarization terms. For pure AIN, the last two contributions are of equal magnitude if $\mathrm{GaN}$ is the substrate [as is often the case in a quantum-well (QW) structure] or, again, the spontaneous polarization is larger if $\mathrm{AlN}$ is the substrate (since $\mathrm{GaN}$ is the well region). For $\mathrm{InGaN}$, the situation is reversed, whereby the spontaneous polarization almost does not contribute; hence, the relatively smaller difference in strain reported by Christmas et al. ${ }^{3}$ is almost entirely due to the difference between the coupled and uncoupled models. In conclusion, the relative importance of the fully coupled model is strongly dependent upon the material system under investigation.

\section{B. Constant electric displacement in space: Monofrequency time dependence}

We next continue with the case where ac conditions apply, i.e., a current $J \propto \exp (i \omega t)$ is led through a piezoelectric film and a three-layer quantum-well structure. Differentiating in time the expression for $S_{3}$ in Eq. (5) and combining with Navier's equation for $T_{z z}$ allow us to write for each material layer,

$$
\begin{aligned}
& \frac{\partial S_{3}}{\partial t}=\frac{\partial v_{z}}{\partial z}, \\
& \rho_{d} \frac{\partial v_{z}}{\partial t}=\frac{\partial T_{z z}}{\partial z}=\left(c_{33}+\frac{e_{33}^{2}}{\epsilon}\right) \frac{\partial S_{3}}{\partial z} \equiv c_{33}^{\prime} \frac{\partial S_{3}}{\partial z},
\end{aligned}
$$

where $v_{z}=\partial u_{z} / \partial t$ is the particle velocity. The latter expression follows as $D$ is constant in space due to Eq. (4) still abandoning cases with free-charge carrier-density distributions. Augmenting $v_{z}$ (respectively, $S_{3}$ ) from Eqs. (21) and (22) yields

$$
\begin{aligned}
& \frac{\partial^{2} S_{3}}{\partial z^{2}}+k^{2} S_{3}=0, \\
& \frac{\partial^{2} v_{z}}{\partial z^{2}}+k^{2} v_{z}=0,
\end{aligned}
$$

with $k=\omega \sqrt{\rho_{d} / c_{33}^{\prime}}$. The general solution for $S_{3}$ and $v_{z}$ due to a current $J=J_{0} \exp (i \omega t)$ are (omitting the trivial factor $\exp (i \omega t)$ in the following)

$$
\begin{aligned}
& S(z)=S_{A} \exp (-i k z)+S_{B} \exp (i k z), \\
& v_{z}(z)=v_{A} \exp (-i k z)+v_{B} \exp (i k z),
\end{aligned}
$$

Consider first a single piezoelectric layer of thickness $l$. If the piezoelectric layer is embedded in vacuum, the boundary conditions $T_{z z}=0$ at $z=0$ and $z=l$ read

$$
\begin{aligned}
& c_{33}^{\prime}\left(S_{A}+S_{B}\right)-\frac{e_{33}}{\epsilon} D=0, \\
& c_{33}^{\prime}\left[S_{A} \exp (-i k l)+S_{B} \exp (i k l)\right]-\frac{e_{33}}{\epsilon} D=0 .
\end{aligned}
$$

These can be solved immediately so as to give (keeping in mind that for dielectric materials the displacement current density fulfills $J=\partial D_{z} / \partial t=i \omega D_{z}$, implying $J_{0}=i \omega D$ )

$$
\begin{aligned}
& S_{B}=\frac{J_{0} e_{33}}{2 \omega c_{33}^{\prime} \epsilon} \frac{e^{-i k l}-1}{\sin (k l)}, \\
& S_{A}=\frac{J_{0} e_{33}}{2 \omega c_{33}^{\prime} \epsilon} \frac{1-e^{i k l}}{\sin (k l)} .
\end{aligned}
$$

Note that the strain coefficients-for this case with no damping mechanisms in consideration-are strongly affected by piezoelectric effects for frequencies nearly satisfying

$$
k l=(2 n+1) \pi
$$

with $n$ an integer. Hence the resonance frequencies are 


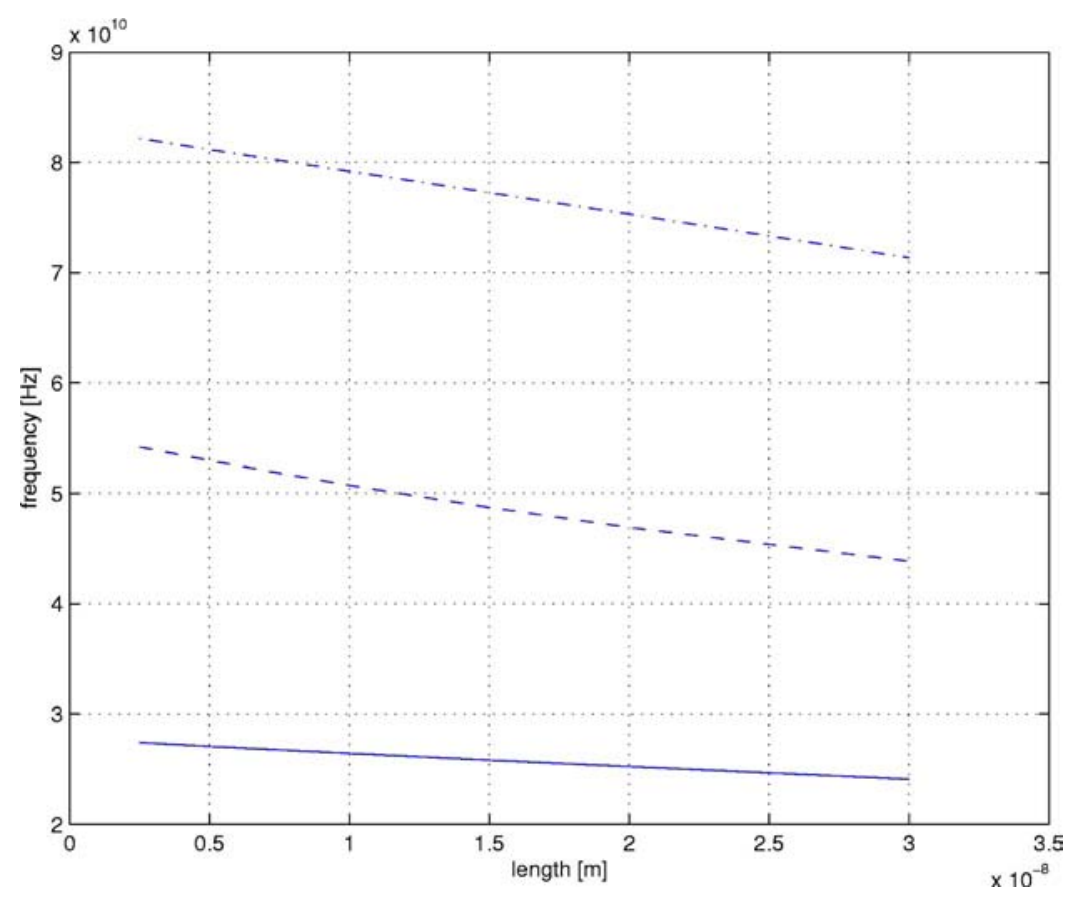

FIG. 3. (Color online) Resonance frequencies of a three-layer quantum-well system corresponding to one layer of GaN sandwiched between two layers of AlN. The figure shows the first three resonance frequencies (solid, dashed, and dashdotted curves, respectively) as a function of the $\mathrm{GaN}$ material-layer lengths. The two AlN layers have the same length equal to $1 \times 10^{-8}$ independent of the GaN material-layer length. Figure values are in SI units.

$$
f=\frac{\omega}{2 \pi}=\frac{(2 n+1)}{2 l} \sqrt{\frac{c_{33}^{\prime}}{\rho_{d}}} .
$$

This result is interesting as it shows that under ac conditions, it is clearly not possible first to determine the strain distributions by minimizing strain energy only (as is done in Refs. 1 and 2) and then calculate-without feedback-the electric fields. We point out here again that the work presented in Refs. 1 and 2 apply well for the case considered in Refs. 1 and 2, namely, corresponding to zero-electric displacement boundary conditions or absence of external electric fields.

We also examine the ac problem for the same three-layer quantum-well structure considered in the first part of this section ( $\mathrm{GaN}$ layer sandwiched between two layers of AlN). Imposing continuity of stress and particle velocity at $z=z_{1}$ $=0$ and $z=z_{2}$ together with vanishing stress at boundaries facing vacuum $\left(z=z_{L}\right.$ and $\left.z=z_{R}\right)$ leads to

$$
\begin{aligned}
& 0=c_{33}^{\prime(1)}\left(S_{A_{1}}+S_{B_{1}}\right)-\frac{e_{33}^{(1)}}{\epsilon^{\|, 1}} D \\
& c_{33}^{\prime}{ }^{(2)}\left(S_{A_{2}}+S_{B_{2}}\right)-\frac{e_{33}^{(2)}}{\epsilon^{\|, 2}} D=c_{33}^{\prime(1)}\left[S_{A_{1}} \exp \left(-i k_{1} l_{1}\right)\right. \\
& \left.+S_{B_{1}} \exp \left(i k_{1} l_{1}\right)\right]-\frac{e_{33}^{(1)}}{\epsilon^{\|, 1}} D \\
& \sqrt{\frac{c_{33}^{\prime(2)}}{\rho_{d}^{(2)}}}\left(S_{A_{2}}-S_{B_{2}}\right)=\sqrt{\frac{c_{33}^{\prime(1)}}{\rho_{d}^{(1)}}}\left[S_{A_{1}} \exp \left(-i k_{1} l_{1}\right)\right. \\
& \left.-S_{B_{1}} \exp \left(i k_{1} l_{1}\right)\right] \\
& c_{33}^{\prime(1)}\left(S_{A_{3}}+S_{B_{3}}\right)-\frac{e_{33}^{(1)}}{\epsilon^{\|, 1}} D=c_{33}^{\prime}{ }^{(2)}\left[S_{A_{2}} \exp \left(-i k_{2} l_{2}\right)\right. \\
& \left.+S_{B_{2}} \exp \left(i k_{2} l_{2}\right)\right]-\frac{e_{33}^{(2)}}{\epsilon^{\|, 2}} D
\end{aligned}
$$

$$
\begin{aligned}
& \sqrt{\frac{c_{33}^{\prime(1)}}{\rho_{d}^{(1)}}}\left(S_{A_{3}}-S_{B_{3}}\right)= \sqrt{\frac{c_{33}^{\prime(2)}}{\rho_{d}^{(2)}}}\left[S_{A_{2}} \exp \left(-i k_{2} l_{2}\right)\right. \\
&\left.-S_{B_{2}} \exp \left(i k_{2} l_{2}\right)\right], \\
& 0=c_{33}^{\prime(1)}\left[S_{A_{3}} \exp \left(-i k_{3} l_{3}\right)+S_{B_{3}} \exp \left(i k_{3} l_{3}\right)\right]-\frac{e_{33}^{(1)}}{\epsilon^{\|, 1}} D .
\end{aligned}
$$

In the expressions above, suffix 1 (2) for material parameter values refers to AlN (GaN) material layers, while $l_{1}, l_{2}$, and $l_{3}$ are the lengths of the first AlN, second GaN, and third AIN layers, respectively. For a given current amplitude $J_{0}$, the electric displacement $D$ equals $J_{0} /(i \omega)$, and the strain coefficients $S_{A_{i}}$ and $S_{B_{i}}$ for each layer $i$ are determined by solving the system of algebraic equations given in Eq. (33). A similar analysis can be carried out for the case where the three-layer structure is embedded in another material.

In Fig. 3, we give the first three resonance frequencies [where the absolute value of the determinant for the coefficient matrix (multiplying the strain coefficients $S_{A_{i}}$ and $S_{B_{i}}$ ) vanishes] for the three-layer structure with AIN layer lengths $l_{1}=l_{3}=1 \times 10^{-7}$, while the GaN layer length $l_{2}$ varies in the range of $2.5 \times 10^{-9}-30 \times 10^{-9}$. We use values for the mass density of $\operatorname{AlN}\left[\rho_{d}^{(1)}=3260\right]$ and $\operatorname{GaN}\left[\rho_{d}^{(2)}=6150\right]$ as in Refs. 7 and 8, respectively. The precise values of the strain coefficients $S_{A_{i}}$ and $S_{B_{i}}$ for a given current near resonances are albeit large not infinite due to acoustic and dielectric losses present in the system.

\section{CONCLUSIONS}

A one-dimensional model accounting for the combined and self-consistent influence of strain and electric fields including contributions from spontaneous polarization, piezoelectric effects, and lattice mismatch was derived. A threelayer AlN/GaN quantum-well structure was solved analytically for the electromechanical-field distributions. It 
was shown, in the absence of current through the structure, that strain distributions depends strongly on both the spontaneous polarization and piezoelectric effects. Furthermore, in cases with ac conditions, resonance frequencies also exist where piezoelectric coupling contributions lead to large strain contributions.

\section{ACKNOWLEDGMENTS}

The work was supported by an NSF CAREER award (NSF Grant No. 0454849), and by a Research Challenge grant from Wright State University and the Ohio Board of Regents. In particular, three of the authors (B.L., R.M., and
M.W.) would like to thank the hospitality of Wright State University where this work was initiated.

${ }^{1}$ V. A. Fonoberov and A. A. Balandin, J. Appl. Phys. 94, 7178 (2003).

${ }^{2}$ V. A. Fonoberov and A. A. Balandin, J. Vac. Sci. Technol. B 22, 2190 (2004).

${ }^{3}$ U. M. E. Christmas, A. D. Andreev, and D. A. Faux, J. Appl. Phys. 98, 073522 (2005).

${ }^{4}$ I. P. Ipatova, V. G. Malyshkin, and V. A. Shchukin, J. Appl. Phys. 74, 7198 (1993)

${ }^{5}$ B. Jogai, J. D. Albrecht, and E. Pan, J. Appl. Phys. 94, 3984 (2003).

${ }^{6}$ H. Liang, M. Upmanyu, and H. Huang, Phys. Rev. B 71, 241403(R) (2005).

${ }^{7}$ G. A. Slack, J. Phys. Chem. Solids 34, 321 (1973).

${ }^{8}$ J. C. Cao and X. L. Lei, Eur. Phys. J. B 7, 79 (1999). 